\title{
Lonza Error Prevention System (EPS) - Changing Human Performance in Pharmaceutical Operations
}

\author{
Kerstin Bodmannª, Constanze Reinhard ${ }^{a}$, Michael Mödlera*, Kevin Tinson ${ }^{\mathrm{b}}$, and Mark Johnson ${ }^{\mathrm{c}}$
}

\begin{abstract}
Errors are a part of life. With human errors accounting for approximately $50 \%$ of quality incidents and related problems within the pharmaceutical industry, the need to improve human performance in manufacturing operations is obvious. The purpose of this article is to describe error-proofing ways of structuring and writing knowledge documents, procedures, batch records, as well as practices for structuring, conducting, and documenting training to assure competence. These practices are recommended for adoption to shift the current 'training for compliance' paradigm to a 'training for competence' paradigm. It will also be demonstrated that a training for competence focus achieves GMP compliance. Results at Lonza have been encouraging, with human error-related quality deviations and non-conformities reduced by more than $40 \%$ across 13 sites globally within the first two years of the implementation of its Error Prevention System.
\end{abstract}

Keywords: Error prevention · GMP · Human performance · Procedures · Training for Competence

\section{Introduction}

Human performance is a key factor for business sustainability, acknowledging that around half of all process-related deviations across the biopharmaceutical industry are currently attributed to human errors, ${ }^{[1]}$ which impact safety, quality, and security of supply.

People are fallible as a part of the human condition, ${ }^{[2]}$ so simply more retraining or disciplinary actions will not necessarily lead to increased performance. ${ }^{[3]}$ Consequently, systematic error prevention is not about a program on top of existing systems, but about changing the way the individual, the leaders, and the organization deal with human errors, including ensuring an open reporting and learning culture.

Beside cultural aspects, clear, simple and accurate procedures are prerequisites for prevention of human errors. A Parenteral Drug Association (PDA) survey found that a typical pharmaceutical company manages an average of 1250 cGMPrequired SOPs with an average document management workload estimated at 10

${ }^{\star}$ Correspondence: Dr. M. Mödler ${ }^{\mathrm{a}}$

E-Mail: michael.moedler@lonza.com

aLonza AG, Lonzastrasse, CH-3930 Visp, Switzerland

bonza Biologics plc, 640 Ajax Avenue

SL1 4DH Slough, UK

'BioPhorum Operations Group (BPOG)

Westbrook Court, Sharrow Vale Road

Sheffield, S11 8YZ UK
$15 \%$ of total operational cost. ${ }^{[4]}$ Based on these costly and time-consuming efforts, one would expect that quality of procedures and documentation systems would be good; however, in general, procedures are not clear, simple, accurate or userfriendly, and systems are not well designed or managed. ${ }^{[5]}$ In addition, training within the biopharmaceutical industry for manufacturing operators often relies heavily on documents written for purposes other than training, such as procedures. A focus on being compliant with having 'read and understood' procedures creates both a large volume of training requirements (both initial and revision training) and acts as a barrier to training for competence. Also, current training structures often lack clarity in what training is required to perform certain tasks.

The impact of this paradigm for training within the industry is that time is spent being 'compliant' with non-value-adding reading requirements, rather than on learning and assuring technical proficiency. Time is spent to maintain large numbers of read-only training requirements that focus on approaching training as a "check the box' activity, rather than learning to competently perform the task. There is also the potential for insufficient and inconsistent technical training, as procedures do not (and should not) contain information required for a performer to learn. All of this leads to a potential for increased errors, since focus is not placed on assuring the level of technical expertise required to operate in a dynamic and complex manufacturing environment. Beyond technical expertise, behavioral and cultural compo- nents of working effectively are also often insufficiently emphasized when procedural documents form the foundation of the learning program. "The training program that brings an individual into compliance does not provide the technical skills needed for his or her role". ${ }^{[6]}$

Learnings within the BioPhorum Operations Group (BPOG) on Human Performance have reinforced the critical importance of technical learning. Another aspect is being adaptive when making decisions or problem solving, by understanding what to do and not to do in a crisis situation. In our dynamic and complex environments, people in their normal work are placed in positions in which they continually need to adapt and make decisions based on changing work conditions. How do we ensure they are prepared to make these decisions? By setting the expectation for, and providing the opportunity for, them to truly learn.

\section{The Lonza Error Prevention System}

To advance human performance towards the next level, Lonza built their global Error Prevention System (EPS). Similar to 'Production Systems' in the automotive industry, Lonza built EPS on the backbone of their Operational Excellence program, adding human performance principles from BPOG immersion meetings around the nuclear and aviation industries' human performance management systems and procedures.

Deployment of the EPS cultural change program is a phased-in, multi-year 
process (Fig. 1). It starts by encouraging everyone to identify pitfalls, habits, and situations that may cause errors and then take actions needed to 'prevent' those errors from happening. The EPS principles and related shop-floor observational coaching tools are supported by a full-day training for all employees and managers. Lonza's approach and the positive results of EPS Phase 1 have been published previously. ${ }^{[1]}$

\section{Effective Documents}

Phase 2 of Lonza's EPS focuses on documents and learning; it started in June 2014, with reviewing external best practices in the areas of documentation and learning from across the industry (facilitated by the BPOG) as well as internal best practices from several Lonza sites.

From an operational perspective there are documents to perform a task and documents to train a task. Documents to perform are those documents that are either referenced or in-hand while an activity is being done. Learning and qualification modules are created to facilitate learning and are generally not used during activity performance. Guidelines and templates for the document types in Fig. 2 were designed by the EPS team accordingly.

Knowledge documents describe policies, process overviews and answer why things are done the way they are done. They include roles and responsibilities, organization and decision governance, environment and regulatory constraints as well as descriptions of strategies. These types of documents may include those that are labeled Policies, Quality Manual components, Quality Directives, and Process Overviews. Job Descriptions that specify roles and responsibilities are considered knowledge documents as well. A sample table of contents for a processrelated knowledge document is given in Fig. 3.

Working documents describe how to do the work; how to perform activities. Working documents will generally need to be in-hand at the time of performance and should have a very small number of pages. These types of documents may include those that are typically labeled work instructions, standard operating procedures, specifications, guides, and job aids. Essential for these documents is to break down tasks into small steps, with each sub-step written in simple language using short sentences and with integrated pictures to further clarify instructions and remove vague guidance. Lists of pre/post job checks, materials and tools required are additional ele-

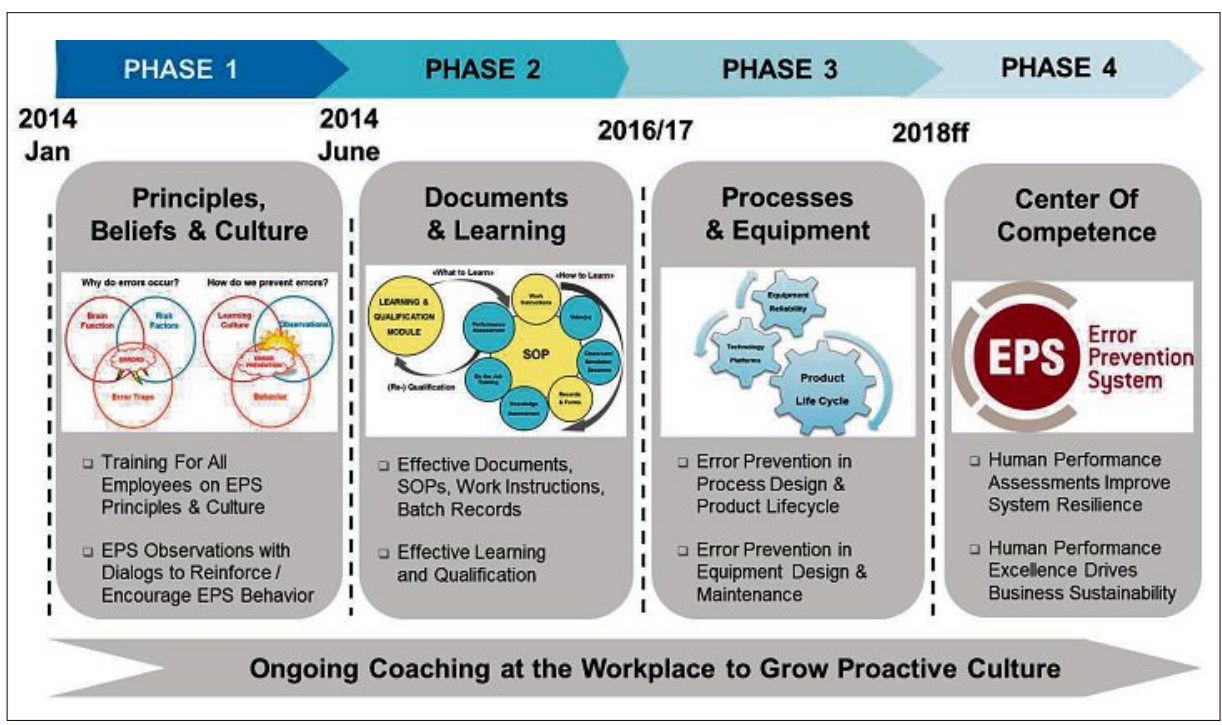

Fig. 1. The four phases of Lonza's Error Prevention System (EPS).

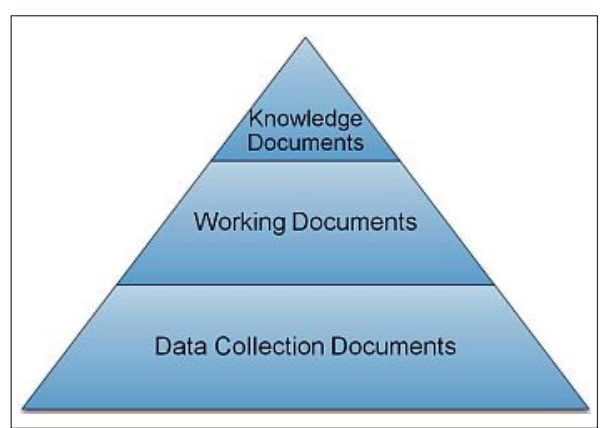

er through the form and avoid omission of steps, standardized symbols and short but precise instructions are grouped in blocks, with references to work instructions as appropriate. Task blocks are surrounded by approx. 30\% of white space, and safety information is listed at start of the block with GHS symbols on hazards and personal protective equipment. A batch record layout example is given in Fig. 5.

Fig. 2. Document types.
Fig. 3. Example table of content for a process-related knowledge document. ments of a typical work instruction, as provided by the example in Fig. 4.

Data Collection documents capture real-time evidence that work was done per instruction as required for traceability. These documents are used during activities to record data, document equipment readings and perform calculations. These types of documents may include those that are labeled forms, batch records, worksheets, and logbooks. To ensure required information is documented, non-entry areas are shaded, and boxes are used for digits with clear specifications and units. To visually guide the perform-

\section{Effective Learning}

Within the industry, utilizing procedures as the base unit for training tends to be the standard approach. This has led to the thinking in some organizations that the compliance requirement is that you are trained specifically by reading and understanding documents. The latest human performance management systems recognize, however, that GMP expectations for training and performance, ${ }^{[7]}$ summarized below, can be satisfied in a variety of ways; one of which is the competencybased approach: 


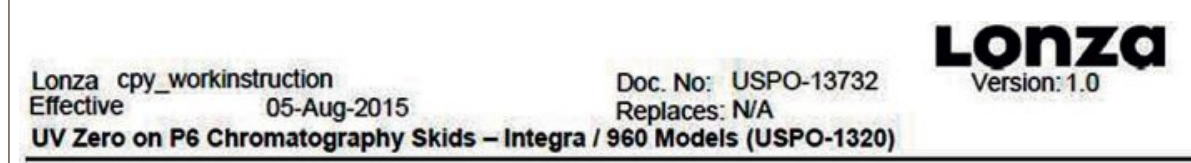

\section{PROCEDURE: EasyCal UV CHECK}

1. Loosen top set screws $\sim 1 / 2$ turn counter-clockwise.

2. Turn $\mathrm{Hi}$ and Lo indicator screws clockwise towards OUT position until screw stops.

3. Hand-tighten top set screws. 3.1 Do not over-tighten.

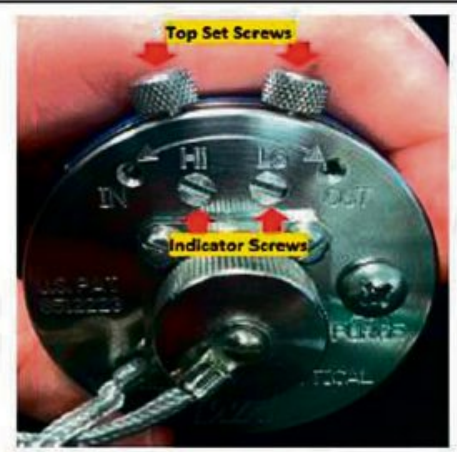

5 PROCEDURE: BASELINE ZERO FOR MODELS INTEGRA, UV 960, 960

1. Press LEFT arrow once to go to PROCESS CONTROL screen.

2. Press DOWN arrow to highlight BASELINE.

3. If UPDATE not displayed, go to UPDATE using RIGHT arrow.

4. PresS Ack/ENT.

5. At Prompt "Are you sure?", press Ack/ENT.

6. Press Esc to return to Process Screen.

Fig. 4. Example of a work instruction.

\section{WI}

6.21 X-27100 Skid Drain

Perform X-27100 Skid Drain per WI USPO-13704.

In the BATCH ID' box enter batch record number, batch record Lot Number and Step Number (USPO-8279/LOTXX_XXXXXX/STEPX.X)
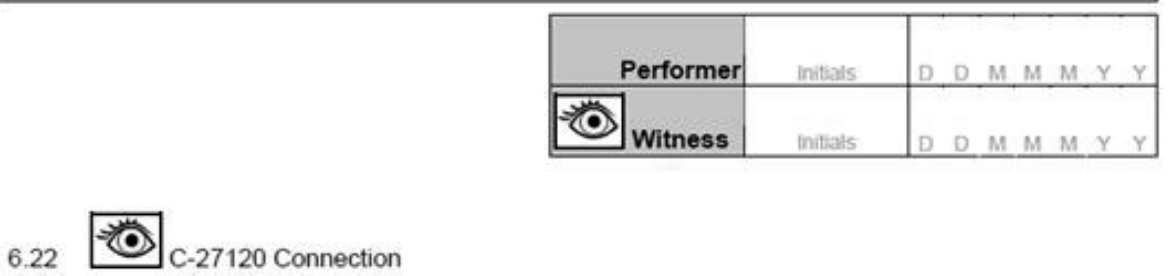

Is mobile column C-27120 connected to chromatography skid?

Select one option:

\begin{tabular}{|l|l|}
\hline$\square$ Yes & Proceed to Step 6.23 \\
\hline$\square$ No & Connect column per SOP USPO-10170 and proceed to Step 6.23. \\
\hline
\end{tabular}

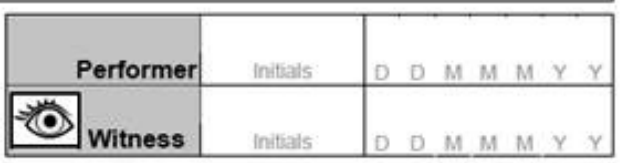

6.23 -

Update $X-27100$ Status Tag to IN USE.

In Use Date/Time
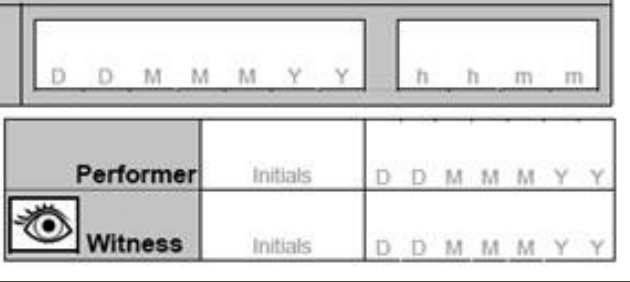

Fig. 5. Example of batch record design.
- There are an adequate number of qualified people to safely and effectively perform the required tasks.

- Tasks, roles and responsibilities are defined in job descriptions and organization charts.

- Personnel are trained in the procedures and methods they use and in the tasks they perform.

- Personnel are trained in the GMP concepts and regulations that apply to what they do.

- Key personnel (including consultants and contractors) have the professional, educational, and experimental credentials required.

- Training is defined by a procedure, an on-going plan, and is documented.

- Training is conducted by qualified personnel.

- Supervisors and management have training that is appropriate to their functions.

Consequently, it is not enough to know what and how to perform a task but the why has to be included too. Also inspectors are increasingly looking for operators to know the why and what happens if something goes differently than expected. In addition, though not necessarily explicit in documented regulations, it is logical for a training program to be able to address questions such as "how do you know personnel are and remain competent to perform their jobs?", and "Is your learning system contributing to quality, compliance and knowledge management?" A training approach that emphasizes "read and understand" as a primary modality most likely will not provide sufficient evidence to address these questions.

Learning activities involve transfer of knowledge, skills and behaviors that affect overall performance. Training on learning activities rather than relying on reading working documents for training provides for increased consistency in learning, more meaningful training and increased knowledge retention.

Fig. 6 illustrates a typical training for competence progression. This training progression starts after an operator joins the organization. Though not discussed in detail in this article, assessments of aptitudes and fit-for-role can be done as part of the recruitment and hiring process, ensuring a good start for new trainees. In application, a learner is considered independently capable to perform following the 'Qualification to Perform' stage.

Learning and qualification modules are designed to summarize performance objectives and provide structure to enable learners to progress towards achieving them. The included training materials support learning the knowledge, skills and 


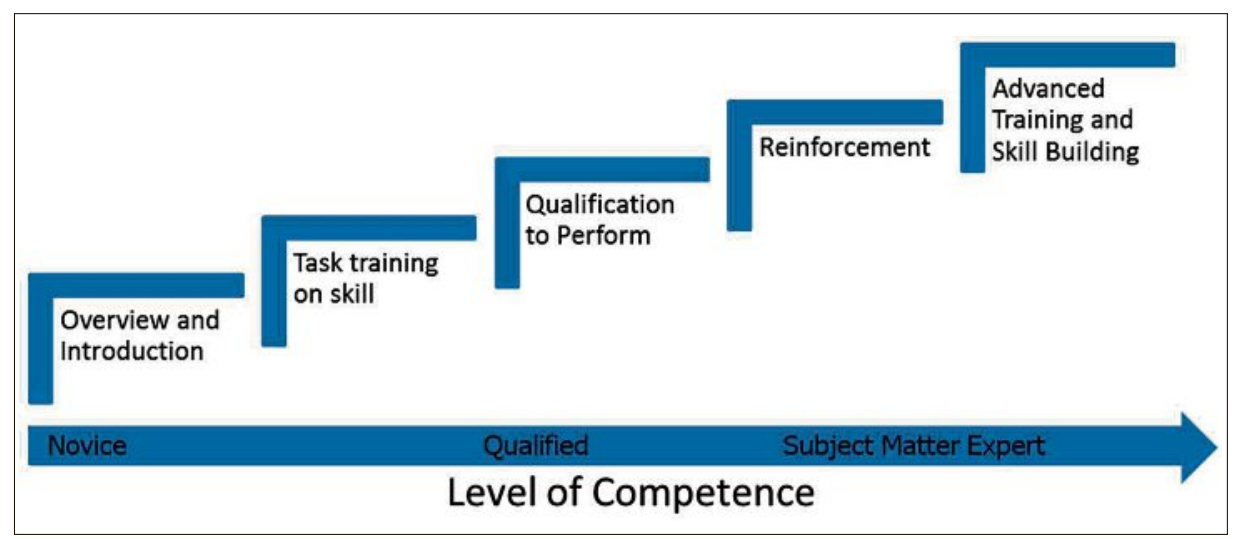

Fig. 6. Training progression.

behaviors required for high levels of performance.

Skills and concepts are initially introduced to the trainee using methods such as seminar led, e-learning, one-on-one training or experimental learning in safe environments. After the initial introduction, the trainee will be trained on basic steps, allowing them to perform in GMP conditions (operating equipment and performing basic steps) while gaining the experience to become proficient. More information, theory and the 'whys' are provided as the trainee progresses through the steps toward being competent in the skill. As the learner continues to advance, he or she learns trouble shooting and advanced operations. Each of these trainings are defined by course plans and documented upon completion. Using this stepwise introduction to build towards competency not only leads to proficiency but also confidence.

The EPS team designed task-specific learning packages to bundle documents and materials for training as well as standardized questions for guidance and performance assessment. These so-called Learning \& Qualification Modules are established by technical experts and operators. For standardization and minimization of administrative work, the same learning packages for sites with the same processes should be used, leading to overall reduced complexity and administrative effort while maintaining compliance. An example on grouping of general learning modules and short training sessions on specific differences for a new test method is displayed in Fig. 7.

Valuable training materials are shop floor videos, as they clearly show how the task has to be done. Operators find these visual lessons easy to grasp, and they avoid the need for pages of written detail to describe what is so easily shown in a video. Videos also ensure consistency in training and are reference for standardized qualification as well as regular assessments on task execution. Key elements of a task-related learning and qualification module are refresher \& requalification requirements, a

Fig. 8. Extract from a learning module.
Upon task assignment, a trainee receives the learning package defined in the corresponding learning and qualification module and gets a qualified operator assigned as mentor. The trainee takes the initiative to learn within a given time-frame by working through the package, accompanied by theoretical knowledge tests and corrective feedback provided by the mentor. Upon completion of all elements within the package, practical competency and theoretical knowledge required for the task are tested by an independent assessor for certification, using standardized questions \& practical demonstrations. The concept is displayed in Fig. 10.

list of references to gain knowledge, guidance and questions on relevant task knowledge, and identification of critical knowledge (Fig. 8). The standardized assessment entails the type of qualification, assigned mentors \& trainers, standardized questions to assess theoretical knowledge, and an independent assessor to verify task execution, following a task-specific checklist. If all elements of the task are carried out as expected and knowledge is as required, a trainee is signed off and qualified to perform the task (Fig. 9).

\section{Implementation}

EPS is embedded as a key element in Lonza's global quality strategy. EPS experts, site leaders, quality assurance and operations people are working jointly to map processes, identify critical steps related to human performance, ${ }^{[8]}$ associated documents, and automation steps as displayed in Fig. 11. This uncovers opportunities not only to standardize and improve tasks, resulting in reduction in

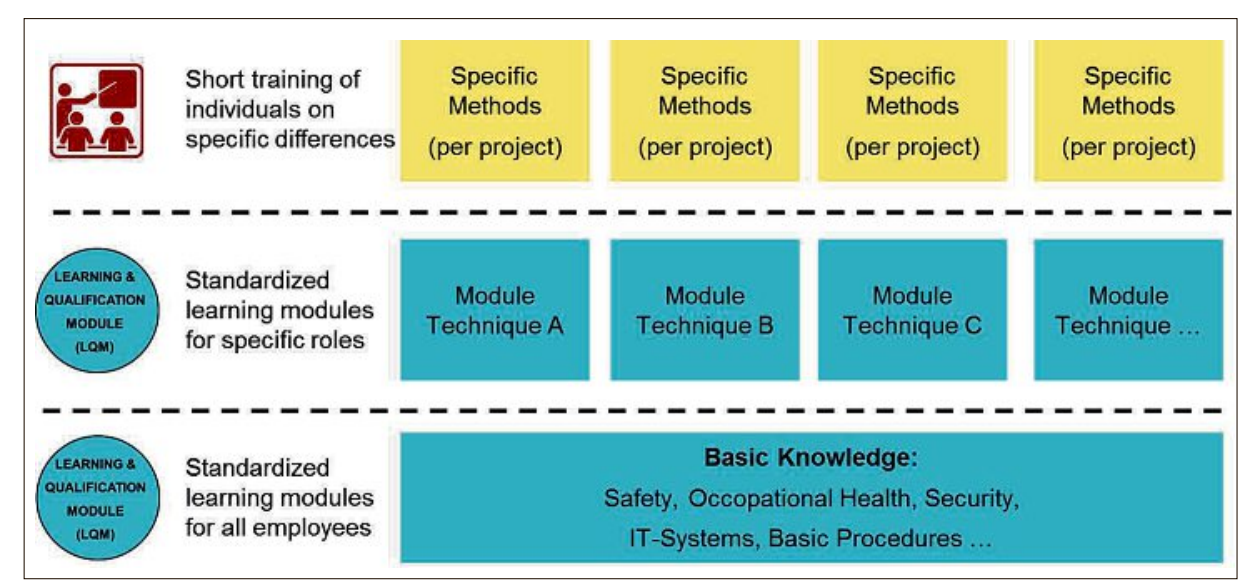

Fig. 7. Example for consolidation of training for competence modules.

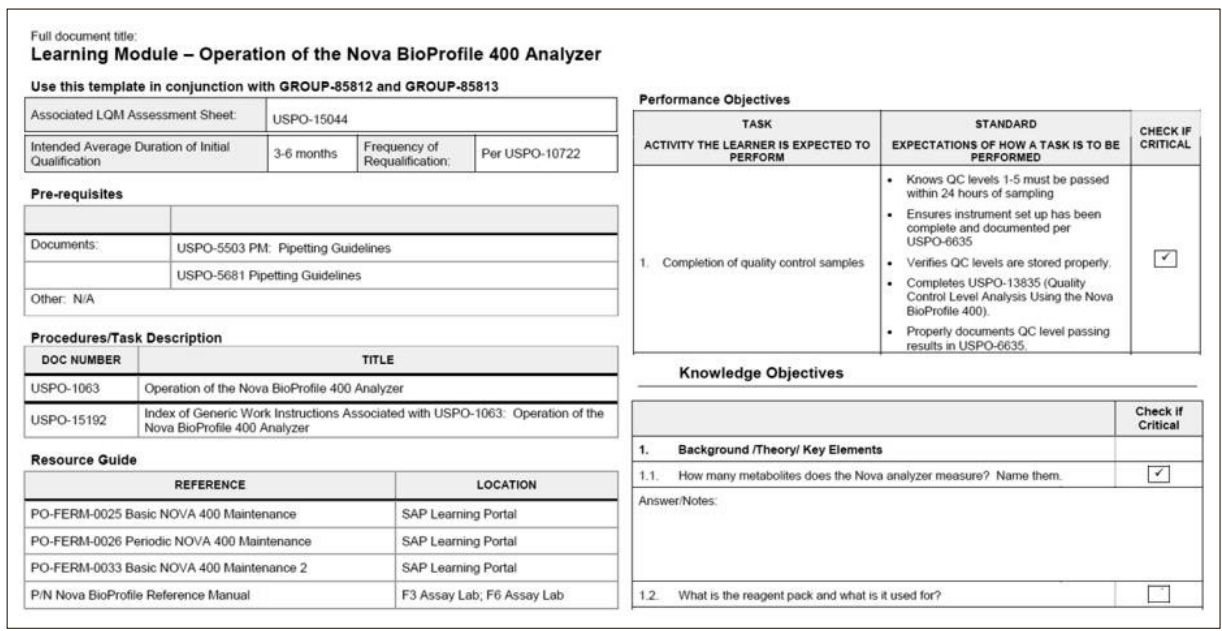




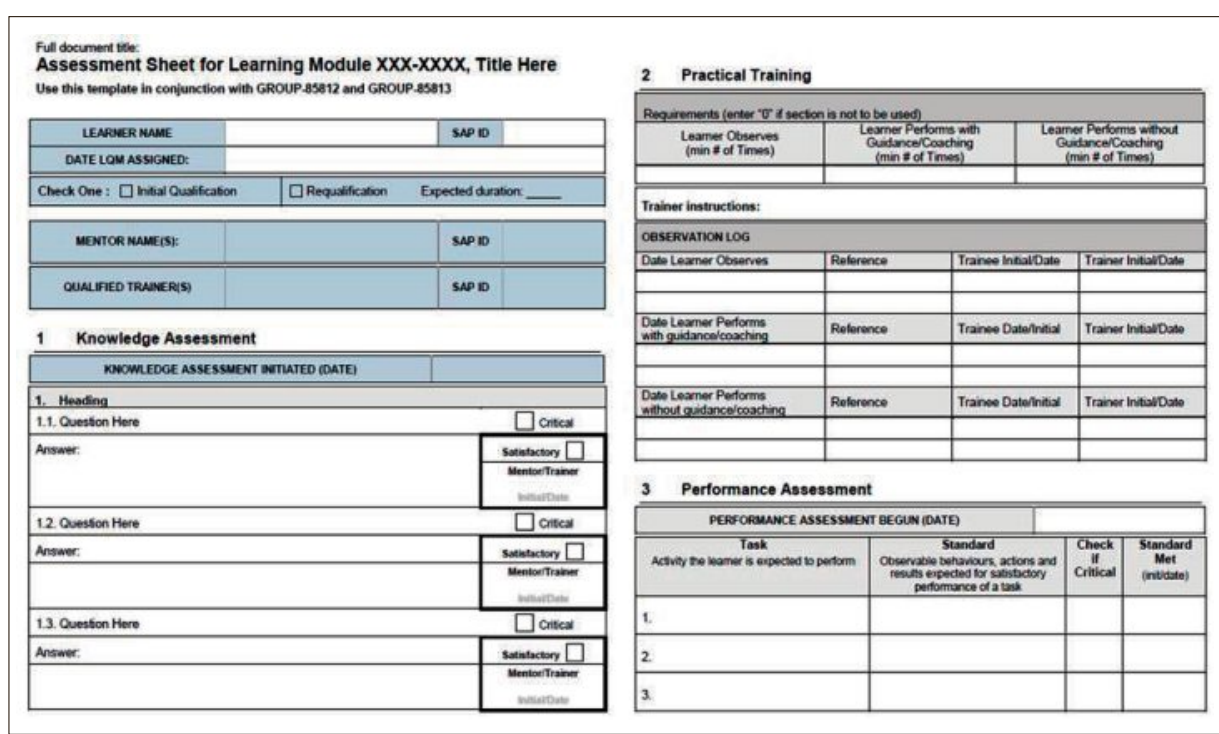

Fig. 9. Elements of a qualification module.

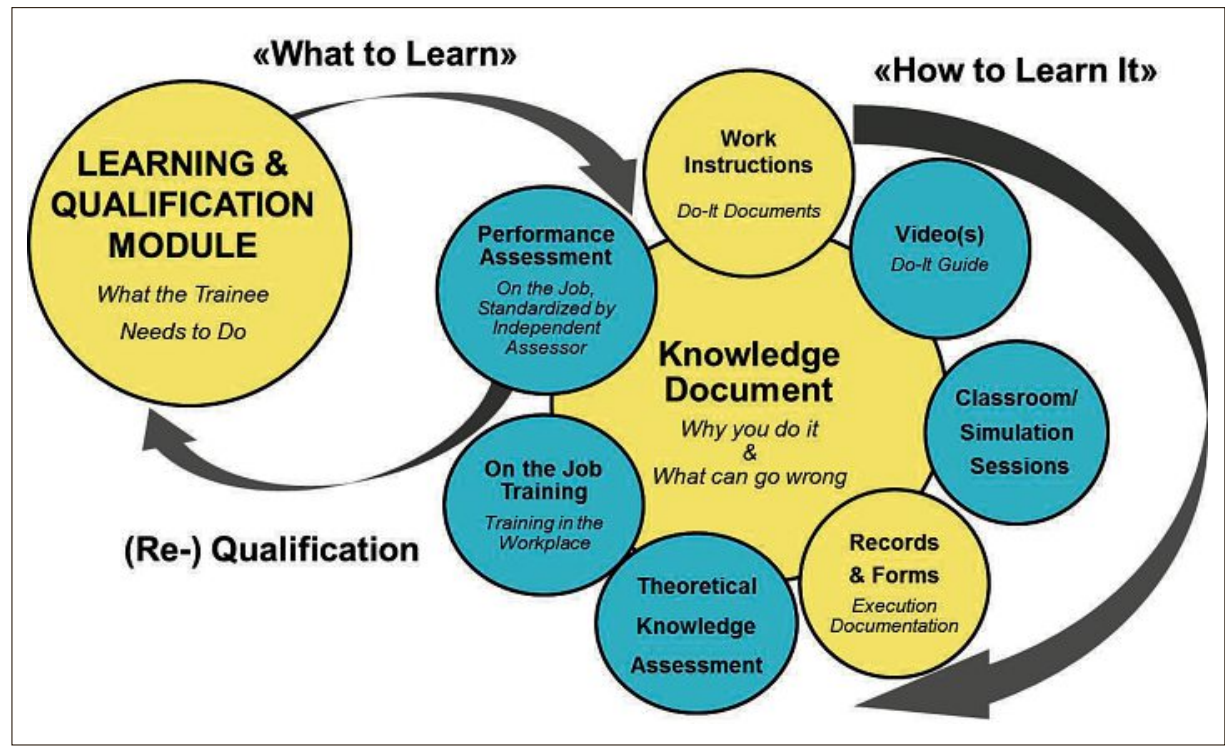

Fig. 10. Example of training for competence. chemical manufacturing in Visp as part of this project are:

- up to $60 \%$ reduction in number of pages for batch records

- up to $50 \%$ reduction in number of entries per batch record

- up to $70 \%$ reduction in number of double checks per batch record

- up to $90 \%$ reduction in number of deviations related to documentation in batch records

One reason for this massive reduction is attributed to a lack of human factor consideration in root cause investigations in the past. Corrective and preventative actions (CAPA) require careful investigation in presence of human errors, otherwise measures like retraining, additional double check or comments in procedures and records are implemented, which inflate documents without being effective as they are mostly not addressing the real root causes.

Implementation of the document concept at commercial scale in a more complex biopharma manufacturing process at another Lonza site achieved reduction of up to $80 \%$ in average number of total deviations per batch (Fig. 12).

Learning and qualification modules (LQM) were piloted at QC Visp on competencies relevant to many operators like 'iLAB', a holistic QC system to foster 'data integrity', eliminate paper, automate processes and ensure procedural execution. Feedback from more than 200 trained operators so far has been very encouraging. For the introduction of iLab, a newly developed LQM was embraced by the operators and knowledge transfer was at a level that no manager on duty was required to answer unclear topics. This approach has reduced failures, such as wrong dilution, overall number of documents, but also to upgrade knowledge documents, simplify work instructions and ensure the sequence and content of steps is reflected correctly throughout all documents.

\section{Results}

A deep dive into deviation categories showed for Lonza's pharmaceutical manufacturing operations in Visp that around one third of all deviations are linked to lapse/memory gaps, error in decision, unclear instructions or inconsistent execution. A Lean Six-Sigma project that was launched around implementation of work instructions for standardized unit operations and batch records with error-proofing formatting as well as simplified, short wording resulted in significant reduction of document complexity at the shop floor. Achieved improvements at large-scale

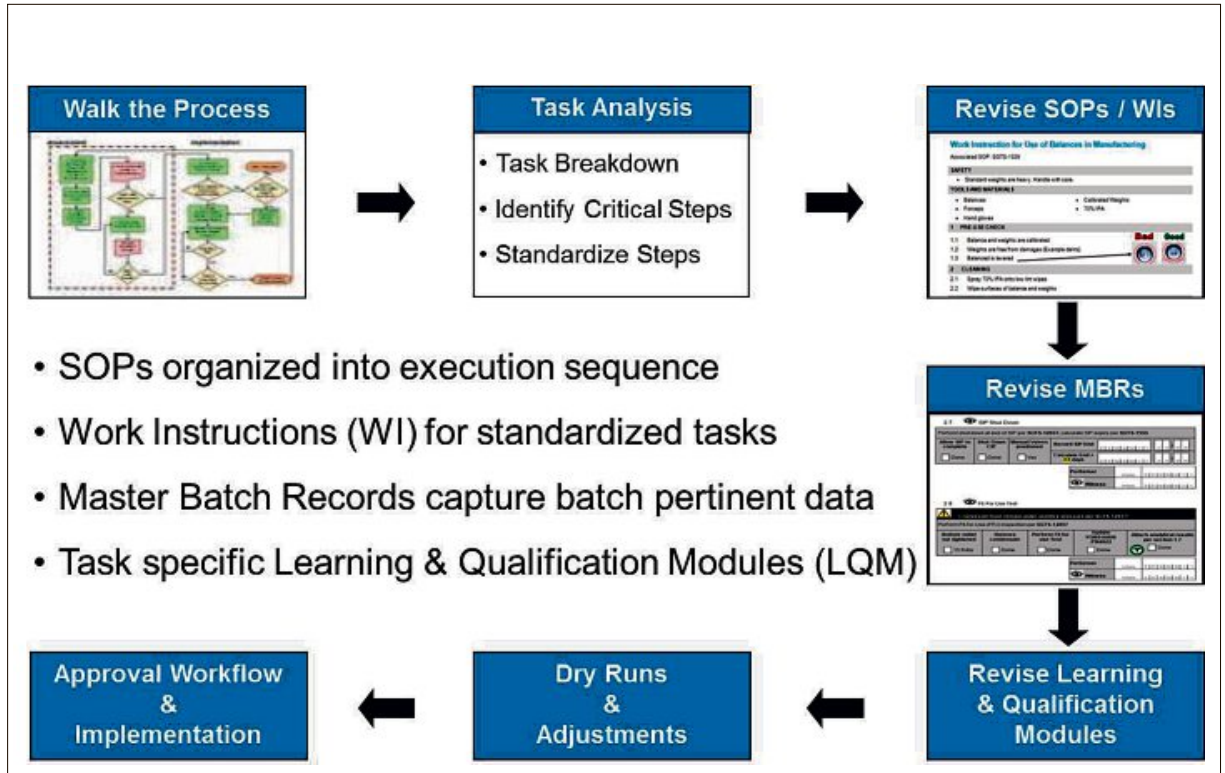

Fig. 11. Workflow for effective documents and learning approach. 


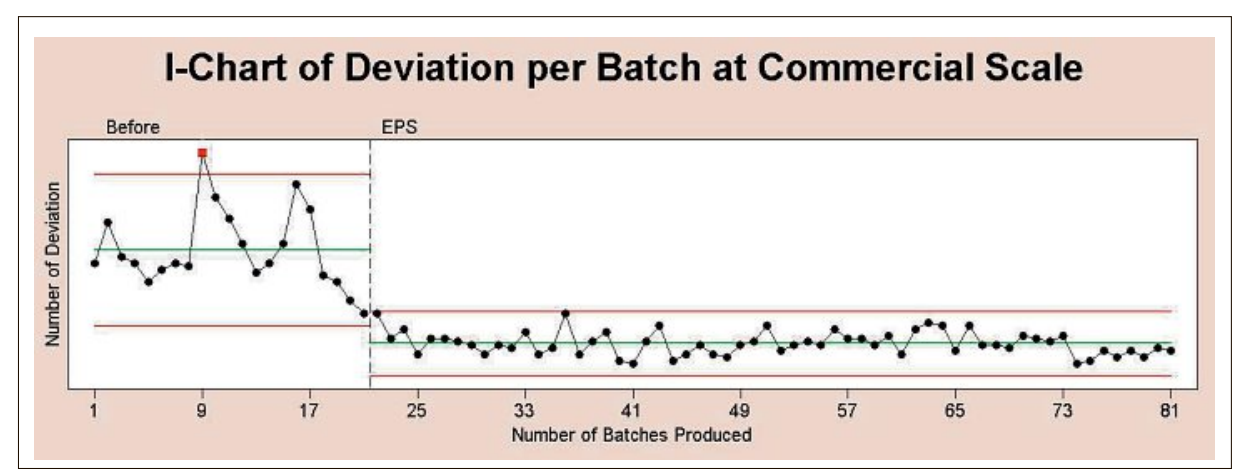

Fig. 12. Reduction in overall deviations per batch by redesign of manufacturing documents.

expired raw materials or overdue standards used, of approximately $20 \%$. Operators are excited to be involved in the development and introduction of further LQMs. A new operator in aseptic working stated that the questions in the corresponding learning and qualification module were perfect to guide him through the key elements of the task and to understand what really matters.

Of course, there are complications with these types of metrics - you'll never know what, or how much you prevent from going wrong. It's difficult to measure the absence of issues, and it's not always straightforward. For example, the loss of batches is not solely influenced by human performance - though the rate has improved year on year also from a financial position, but an exact number is difficult to establish.

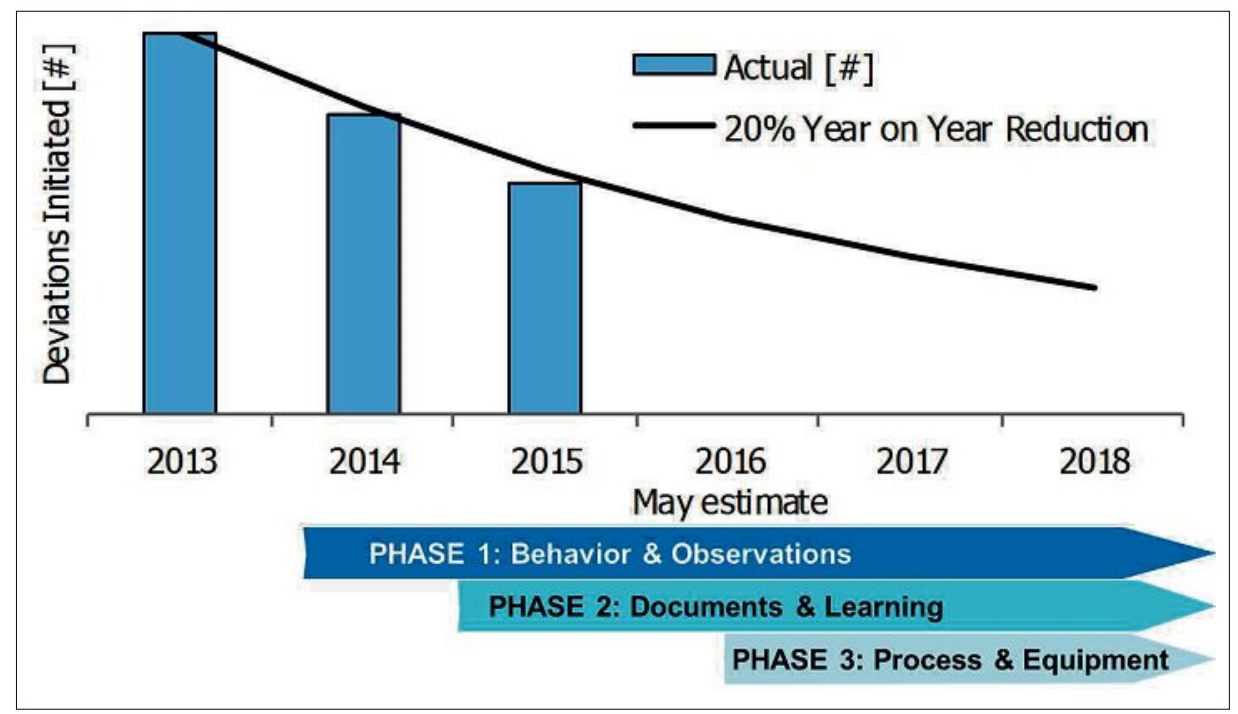

Fig. 13. Development of the number of deviations initiated for Lonza Network.

\section{Conclusion}

Previously, the focus for performance improvement was mainly on technical solutions and controls. Employees and their knowledge were not appropriately involved, and it was hard to get them excited. Now, rather than focusing solely on what was done wrong, we ask ourselves what kind of environment does an operator need to do a good job, turning the whole thing upside down. The bottom line, though, comes through seeing tangible improvements - site to site, and across the organization. There is a lot of evidence that human performance efforts are preventing negative outcomes. Even if results are visible in reduction of operator-error deviations, it is important to move away from labelling errors as the operators' fault, and moving to a more proactive and preventative approach.

The goal of driving a corporate human performance program on error prevention is not just financial, cultural or performance motivated. The ultimate objective is to enhance patient health and safety by manufacturing drug products of the highest quality.

Received: June 8, 2016

[1] A. Wilson, M. Moedler, G. McAuley. PDA J. Pharma. Sci. Technol. 2015, 69, 658.

[2] J. Reason, 'Managing the Risks of Organizational Accidents' Ashgate: Farnham, England, 1997.

[3] S. Dekker, 'Field Guide to Understanding 'Human Error", 3rd ed.; Ashgate: Farnham, England, 2014.

[4] D. Gingell, Pharm. Technol. Europe 2001, 4.

[5] R. G. Kieffer, Pharm. Technol. 2013, 64.

[6] K. M. Kapp, 'A Tale of Two C's: From Compliance to Competency', 2015 uleduneering.com.

[7] J. Vesper, 'GMP in Practice', 4th ed, 2011.

[8] T. Muschara, Perf. Improv. 2014, 53, 11. 